Théologiques

Théologiques

\title{
Danser avec les esprits
}

Explorations de l'univers amérindien

\section{Achiel Peelman}

Volume 2, numéro 2, octobre 1994

L'esprit

URI : https://id.erudit.org/iderudit/602408ar

DOI : https://doi.org/10.7202/602408ar

Aller au sommaire du numéro

\section{Éditeur(s)}

Faculté de théologie de l'Université de Montréal

ISSN

1188-7109 (imprimé)

1492-1413 (numérique)

Découvrir la revue

\section{Citer cet article}

Peelman, A. (1994). Danser avec les esprits : explorations de l'univers amérindien. Théologiques, 2(2), 73-90. https://doi.org/10.7202/602408ar
Résumé de l'article

Un des aspects les plus frappants de la réalité multiculturelle et plurireligieuse du Canada est la persistance de l'univers spirituel des Amérindien(ne)s. Cet article offre un aperçu et une interprétation de la place des " esprits » dans ce monde autochtone, contemporain. Au moyen de la description d'un rituel particulier (la suerie), nous essayons de saisir la nature et la fonction des entités qui sont désignées comme " esprits ", " puissances " ou "puissances spirituelles ", tout en soulignant l'actualité et la valeur thérapeutique de la médecine amérindienne, traditionnelle. d'utilisation que vous pouvez consulter en ligne.

https://apropos.erudit.org/fr/usagers/politique-dutilisation/ 
Théologiques 2/2 (1994) 73-90.

\title{
Danser avec les esprits
}

\section{Explorations de l'univers amérindien}

\author{
Achiel PEELMAN \\ Faculté de théologie \\ Université Saint-Paul
}

\section{RÉSUMÉ}

Un des aspects les plus frappants de la réalité multiculturelle et plurireligieuse du Canada est la persistance de l'univers spirituel des Amérindien(ne)s. Cet article offre un aperçu et une interprétation de la place des esprits $*$ dans ce monde autochtone, contemporain. Au moyen de la description d'un rituel particulier (la suerie), nous essayons de saisir la nature et la fonction des entités qui sont désignées comme "esprits*, "puissances * ou * puissances spirituelles *, tout en soulignant l'actualité et la valeur thérapeutique de la médecine amérindienne, traditionnelle.

Un des aspects les plus frappants de la réalité multiculturelle et plurireligieuse du Canada est la persistance étonnante de l'univers spirituel des Amérindiens. Les religions amérindiennes figurent parmi les religions les plus anciennes du monde ayant réussi à demeurer des religions vivantes. Leur renaissance actuelle ne saurait échapper aux observateurs et observatrices de la réalité autochtone. Emma Laroque, une auteure amérindienne, constatait déjà en 1975: "S'il existe aujourd'hui une renaissance amérindienne, c'est dans le monde de la religion. Les jeunes s'adressent de nouveau aux anciens pour chercher une aide spirituelle. Les anciens commencent à partager de nouveau leurs secrets" (Laroque 1975: 27). Dans cet article nous jeterons un regard discret sur une des composantes majeures de cette expérience religieuse millénaire: l'identité et la signification des entités que les pratiquant(e)s des religions amérindiennes désignent comme «esprits». Il importe de noter que le terme «religions 
amérindiennes" se refêre ici à l'ensemble des pratiques religieuses et des attitudes spirituelles, des rituels, des cérémonies et des croyances qui forment la spiritualité traditionnelle telle que vécue, da l'heure actuelle, par les divers groupes amérindiens $d u$ Canada. Ce article offre un aperçu et une interprétation de la place éminente des "esprits" dans ce monde autochtone, contemporain.

\section{L'univers spirituel des Amérindiens}

\subsection{Les traits fondamentaux de l'expérience religieuse amérindienne}

Les religions amérindiennes se laissent comprendre comme une sorte de "métaphysique traditionnelle de la nature» (Brown 1990) où les frontières entre le religieux et le non religieux, entre le scientifique et le surnaturel, entre le sacré et le profane sont très vagues, au point d'être constamment traversées dans la conscience intime des personnes et dans l'interprétation des événements (Marty 1990: xi-xiv). Cette flexibilité a son origine lointaine dans le fait que les religions amérindiennes n'ont ni fondateur historique ni contenu dogmatique et qu'elles ne sont jamais devenues des religions "institutionnalisées" à la façon des grandes religions historiques. Étant donné que les langues amérindiennes ne possèdent pas de vocables pour exprimer ce que nous entendons habituellement par "religion", "Église», "théologie» ou "philosophie ", nous avons recours à des termes occidentaux pour exprimer l'univers religieux des amérindiens. Le terme "religion(s) amérindienne(s)" peut ête considéré comme l'équivalent des termes «spiritualité amérindienne" ou "médecine amérindienne". Quels que soient les termes que nous utilisons pour cerner l'expérience religieuse amérindienne, celle-ci se présente fondamentalement comme un cheminement spirituel, un processus religieux ou un état de conscience concernant le "Grand Mystère" (Wakan tanka, le nom le plus traditionnel du Dieu amérindien) et concernant les "puissances spirituelles», les «esprits" (Spirits) ou les "puissances" (Powers) de l'univers.

Malgré la grande variété des croyances, des mythes et des rituels par lesquels elles s'extériorisent d'un groupe amérindien à l'autre, les religions amérindiennes reposent sur un certain nombre de facteurs qui se retrouvent pratiquement partout et qui constituent les points de repère indispensables pour bien comprendre et respecter l'univers spirituel dans lequel les Amérindien(ne)s ont évolué jusqu'à l'époque contemporaine. Parmi ces facteurs, il importe de souligner en premier lieu le caractère holistique des religions amérindiennes. Celles-ci ne constituent pas un secteur à part dans les sociétés autochtones (comme c'est le cas de la reli- 
gion chrétienne dans notre monde sécularisé), mais forment, au contraire, le substratum le plus propond et le plus intime de toute la vie amérindienne. Les religions amérindiennes se présentent ensuite comme des religions «expérientielles", "mystiques" et «existentielles». L'expérience constitue le cœur de l'apprentissage amérindien dans tous les domaines de la vie. Les religions amérindiennes reposent sur un grand respect de l'expérience personnelle de Dieu et des puissances spirituelles. Elles ont développé au cours des siècles une sensiblité très vive du "Grand Mystère " qui est partout, tout en étant insaisissable. Black Elk, le grand prophète Lakota, affirmait: "Nous devons bien comprendre que tout est l'œuvre du Grand Esprit. Nous devons savoir qu'il est présent en tout: les arbres, les herbes, les rivières, les montagnes, les animaux et les oiseaux ...» (Brown 1971: xx). Beaucoup d'Amérindien(ne)s se sentent mystiquement lié(e)s aux "esprits" des animaux, des plantes, des arbres, des pierres, de l'eau et de tous les autres êtres vivants. Leur conception de la nature est caractérisée par une "harmonie cosmique» qui se réalise au moyen des justes relations qui s'établissent ou qui doivent s'établir entre tous les vivants de l'univers. La fonction principale de beaucoup de rituels amérindiens est de prendre explicitement conscience de cette "harmonie cosmique». La survie des religions amérindiennes dépend, en grande partie, de la volonté et de la capacité de participation effective des membres de la communauté à leur actualisation. Les religions amérindiennes n'existent que dans la mesure où elles sont pratiquées, ici et maintenant. Le fait que nous disposons aujourd'hui d'un nombre croissant d'études ethnographiques sur elles ne change rien à cette réalité. Les religions amérindiennes reposent sur des personnes qui sont (encore) capables d'entrer en communion avec Wakan Tanka et avec les puissances spirituelles. En effet, ce qui les caractérise fondamentalement, c'est le lien intime entre leur dimension informative et leur dimension performative (Gill 1987), ainsi que leur configuration symbolique ou sacramentelle, très concrète. Ceci accentue, à la fois, le rôle des rituels comme lieu d'apprentrissage pratique ou expérientiel, le rôle des personnes (ancien(ne)s, sages, chamanes, prophètes, rêveurs, guérisseurs) qui ont reçu des dons particuliers dans le domaine des communications spirituelles, ainsi que l'importance des rêves ou des visions qui sont considérés comme les canaux par excellence de cette communication.

\subsection{Une illustration particulière: la suerie}

Depuis 1983 nous avons eu le privilège de participer à un certain nombre de rituels tels que la quête de vision (Vision quest), les rituels du Calumet sacré, les célébrations de commémoration des ancêtres, des 
rituels variés de guérison, et la suerie (Sweat lodge) qui ont été les lieux concrets de notre propre initiation à l'univers spirituel des Amérindien(ne)s. Afin de bien saisir en quoi on s'engage quand on accepte une telle initiation, nous présentons ici la description détaillée d'un rituel de suerie auquel nous avons participé en 1991.

Thunder Bay, le 22 octobre 1991. La nuit est tombee sur la ville. Vers 21 h30, dix hommes quittent silencieusement le confort de leur chambre d'hôtel pour se rendre dans un bois environnant. La neige craque sous leurs pieds. Un vent brusque fait accélérer leurs pas en direction d'une clairiere illuminée par un feu très vif ...

Arrivés à l'endroit, les hommes sont accueillis par le président (medicine man) et son assistant. Chacun pose une pincée de tabac dans le feu où une quinzaine de grosses pierres sont chauffées à blanc. Le président les invite à former un cercle parfait autour du feu. D'un geste solennel, il ouvre son sac-médecine où se trouve son calumet sacré. Il joint le fourneau au tuyau et procède au bourrage rituel du calumet. Entre le pouce et l'index de la main droite, il prend une pincée de tabac qu'il dépose d'un geste précis dans le fourneau. Ce mouvement est répété sept fois en hommage aux esprits des quatre points cardinaux, du ciel et de la terre-mère, et aux messagers divins qui viendront participer à la cérémonie de la suerie qui vient de commencer. Une fois le bourrage rituel terminé, le calumet sacré est déposé sur un monticule de terre qui se trouve entre le feu et une petite cabane circulaire, en forme de dôme, construite par le président et son assistant au cours de la journée.

Les hommes se déshabillent. Sur l'invitation du président, ils entrent l'un après l'autre dans la cabane par une ouverture étroite qui fait face à l'Est. Tous sont assis sur des branches de cèdre, le dos conte la paroi de la cabane, autour du trou situé exactement au centre de la suerie. Le président est assis près de la "porte" de l'Est. À côté de lui se trouve un seau d'eau de source. Il ordonne maintenant à son assistant d'introduire délicatement les pierres chauffées à blanc dans la cabane. Elles sont placées dans le trou du centre. La "porte" de l'Est est maintenant fermée. L'obscurité est totale. Le président "bénit» les pierres en jetant sur elles des branches de cèdre et des herbes parfumées. La cabane se remplit d'une chaleur intense. On n'entent que le sifflement des pierres et la respiration lourde des hommes dont on distingue à peine la sihouette courbée. Une paix profonde s'installe dans la petite cabane.

Le silence est soudainement rompu par les instructions du président. Ce soir, dit-il, on suivra la tradition des Indiens Lakota selon les enseignements qu'il a lui-même reçus de son guide spirituel. La cérémonie se 
tiendra en communion avec les "Vents" ou les esprits des quatre points cardinaux de l'univers. Il y aura quatre rondes de prière en hommage aux quatre «portes»: la porte de l'Est par où sont entrés les humains, et les portes du Sud, de l'Ouest et du Nord (qui ne sont jamais ouvertes) par où entrent les puissances spirituelles qui viendront participer à la cérémonie. Chacun parlera à son tour, aussi longtemps qu'il veut et dans la langue de son choix. Chacun est invité à prier, à chanter, à exprimer ses sentiments profonds, et à s'adresser avec confiance au Grand Mystère (le Créateur). Le président (Monsieur Don Goodwin, medicine man et diacre ordonné du diocèse de Grand Rapids, Michigan) rappelle que le Fils du Créateur, notre frère aîné Jésus, occupe une place unique parmi les puissances spirituelles qui participeront au rituel. Après chaque ronde de prière, on observera un bref moment de repos sans toutefois ouvrir la porte de l'Est. Si quelqu'un devenait malade au cours de la cérémonie, il pourrait alors demander au président la permission de quitter la cabane.

Au commencement de la première ronde de prière en hommage aux esprits de l'Est, le président arrose les pierres abondamment avec l'eau de source. Une vapeur intense, à peine supportable, se dégage immédiatement dans la cabane. Il répétera ce geste au début et à la fin de chaque ronde de prière. Le président commence lui-même chaque ronde en évoquant respectivement les puissances spirituelles (nos esprits-gardiens, "grands-pères" ou "grands-mères") de chaque point cardinal, en rappelant les énergies et les valeurs qu'elles représentent et en partageant avec les autres son propre témoignage ou récit. Il invite ensuite son voisin de gauche à poursuivre la prière.

Les prières et les récits personnels sont aussi intenses que la chaleur qui s'est installée dans la cabane. Tantôt c'est la souffrance et la misère humaine, dans toute leur nudité, commes les corps exposés à la chaleur purifiante des pierres: des récits de violence et l'imploration du pardon. Tantôt c'est une prière d'action de grâce ou un chant de joie: des récits de guérison et de réconciliation. On entend des murmures d'approbation et d'encouragement qui viennent du fond des cœurs. On entend des sons mystérieux qui signalent la présence de puissances spirituelles. Le cercle des prières et des témoignages est complété quatre fois: des prières pour soimême, pour la famille, pour la paix entre les peuples du Canada et du monde entier, pour le bien-être des communautés autochtones et l'avenir des Premières Nations, pour les Églises chrétiennes et les autres religions. On invoque les ancêtres et tous les êtres vivants de l'univers.

En sortant de la cabane, à la fin du rituel, les hommes s'acclament: "Toutes mes relations!". Ils forment de nouveau un cercle parfait autour 
du feu. Ils se tiennent maintenant debout, renouvelés, revitalisés, régénérés. Ils sont rentrés dans le grand cercle de la Vie dont le Grand Mystère est le centre. Ils concluent la célébration en fumant le calumet sacré. La lune veille sur la ville de Thunder Bay. Il est deux heures du matin.

\subsection{Les puissances spirituelles, les puissances et les esprits}

Le rituel que nous venons de décrire ne constitue pas un fait isolé. Un peu partout au Canada nous rencontrons aujourd'hui ce type de célébrations. Elles impliquent un nombre considérable d'autochtones quel que soit leur degré d'acculturation à la société dominante: les "traditionnalistes " (peu affectés par la culture occidentale et le christianisme), les "itinérants culturels" (ceux et celles qui, selon les situations concrètes de la vie, pratiquent la religion traditionnelle ou le christianisme), les "biculturels" (ceux et celles qui ont élaboré une synthèse personnelle des deux systèmes religieux), et les "générations perdues" (ceux et celles qui, coupés de leurs traditions ancestrales, y retournent aujourd'hui, souvent dans le contexte d'une démarche thérapeutique).

Dans tous ces rituels, quel que soit le statut culturel ou religieux des personnes qui les pratiquent, nous constatons un dénominateur commun: la référence explicite aux entités qui sont désignés comme "esprits". Les diverses langues amérindiennes disposent d'ailleurs de leurs propres termes techniques pour nommer ces entités et, dans certains cas, pour signifier leur rapport avec l'Être absolu. Par exemple, chez les Dènè Tha de l'Assomption, au Nord-Ouest de la province de l'Alberta, Echint'e signifie "puissance" (pouvoir; Power; entités spirituelles); la personne qui a rencontré son animal protecteur (pouvoir) s'appelle dene wonlin edadihi (une personne qui connaît un animal); le principal rituel religieux (cérémonie des offrandes), présidé par les prophètes dènè, s'appelle dawots'ethe («on danse») ou Tea Dance en anglais (Goulet 1992). C'est à l'Assomption que nous avons appris à danser avec les esprits! Les Lakota de la réserve de Rosebud (South Dakota), eux-aussi, connaissent plusieurs sortes d'esprits: les Itopta Sapa (chevreuil à queue noire) qui peuvent prédire l'avenir; les Inyan Wasicu (esprits-roches), au nombre de 405, qui sont des esprits protecteurs participant au rituel de la suerie (Yuwipi); les Heyoka (clowns) qui enseignent ce qui est bon ou mauvais en faisant le contraire; les Pehin San (cheveux gris), les esprits de sagesse, et les Iktomi (esprits-araignées; trickster) qui assurent les biens matériels, mais qui peuvent exiger des offrandes considérables (Stolzman 1986: 105-115).

Quand on est confronté sur le terrain avec cette dimension plus mystérieuse de la réalité amérindienne, surtout dans le contexte des rituels 
religieux, on ne peut s'empêcher de s'interroger sur le statut ontologique de ces "esprits". Les Amérindien(ne)s, de leur côté, semblent peu préoccupé(e)s par cette question ontologique ou métaphysique. Face aux "esprits", ils/elles adoptent un point de vue tout à fait fonctionnel, dominé par le souci d'entretenir des relations justes avec ces "êtres spirituels" qui peuvent exercer une influence, bonne ou mauvaise, sur tous les aspects de la vie.

Les théologien(ne)s qui s'aventurent sur ce terrain pourraient se tourner vers la Lettre aux Ephésiens et la Lettre aux Colossiens pour y chercher quelconque éclairage. Nous constatons cependant que les "entités spirituelles» auxquelles refère Paul (Ep 2,2 le chef de la puissance de l'air, les fils de la rebellion; Ep 6,12 Autorités, Pouvoirs, Dominateurs, esprits du mal ; Col 1,16 Trônes, Souverainetés, Autorités, Pouvoirs) sont présentées comme des puissances démoniaques (dont le statut ontologique n'est pas défini non plus - leur noms ne sont que leurs symboles) qui cherchent par leur action à s'emparer de l'être humain et de son univers sous le signe destructeur de la mort (Schlier 1958 et 1968: 171-185; Wink 1984 et 1986). L'univers spirituel des Amérindien(ne)s est plus complexe et l'action des esprits qui y habitent est ambivalente. Dans cet univers, le hasard n'existe pas. Tout est lié à tout. Même si les esprits amérindiens sont généralement perçus sous le signe constructif de la vie dans leur association étroite avec le Grand Mystère, ils peuvent être utilisés par des mauvais medicine men, abusant de leur pouvoir spirituel, pour causer du mal. Nous avons pu constater un peu partout au Canada que les Amérindien(ne)s n'abordent pas facilement ce sujet du bad medicine par crainte de déranger l'harmonie cosmique qui règne dans l'univers des esprits.

\subsection{Wakan Tanka et les esprits}

Une des principales fonctions de toute religion est de nommer l'Être suprême et de produire une vision cohérente de la distribution des puissances spirituelles dans l'univers. Le caractère existentiel, mystique et expérientiel des religions amérindiennes suggère immédiatement que leur connaissance de Dieu se situe de prime abord au cœur de la vie quotidienne. L'Être suprême n'est pas d'abord perçu comme celui qui se trouve à l'origine (protologie) ou à la fin (eschatologie) du monde, mais comme le fondement de tout ce qui est, ici et maintenant. Il est le véritable centre de tout être vivant et "présence" dans l'univers. Faire expérience de cet Être suprême implique donc au point de départ que l'on trouve sa juste place dans l'univers et que l'on établit des relations justes. Cette expérience de Dieu est fondamentalement une question d'harmonie interne et 
externe, parce que l'univers entier est perçu comme une réalité sacrée ou comme le lieu où habitent les puissances spirituelles (Peelman 1992: 69. 78). À cause de cette approche particulière du mystère de Dieu, on a longtemps pensé que les Amérindien(ne)s n'avaient pas de concept d'un Être suprême. Selon la perspective évolutionniste du XIX ${ }^{\mathrm{e}}$ siècle, influencée par la doctrine de l'animisme d'Edward Tylor, religions "primitives" et "monothéisme" étaient des réalités irréconciliables (Evans-Pritchard 1956). Les premiers anthropologues pensaient que la religion évoluait nécessairement du culte de la nature vers le panthéisme, le dualisme, et, ultimement, vers le monothéisme. Aujourd'hui, nous ne remettons plus en question que la religion traditionnelle des Amérindien(ne)s était déjà structurée autour du concept d'un Être suprême lors de son contact avec le christianisme. Mais cette religion traditionnelle ne se laisse pas si facilement enfermer dans les concepts occidentaux du polythéisme et du monothéisme. Le concept occidental qui nous semble le plus apte à exprimer la spécificité de l'expérience religieuse amérindienne est celui du pan-enthéisme, emprunté à la mystique chrétienne. Dieu est tout et, pourtant, audessus de tout. Il n'est pas tout simplement l'Autre, mais le Non-Autre. Les plus anciens noms amérindiens de Dieu, tels que Wakan Tanka ("Grand Mystère") chez les Lakota, témoignent d'une vision tout-inclusive de l'Être suprême. Dans certaines cultures amérindiennes, comme chez les Dine (Navajo), l'Être suprême n'avait pas de nom propre. Il était vénéré comme la Grande Puissance Invisible universellement présente dans la création, qui ne se laissait tout simplement pas définir ou nommer. Avant le contact avec le christianisme, la majorité des cultures amérindiennes semblaient s'opposer aux représentations anthropomorphiques de l'Être suprême. Les Amérindien(ne)s ne cherchaient pas une "relation personnelle" avec Dieu qu'ils/elles percevaient plutôt comme une présence insaisissable et indéfinissable ou comme le fondement de leur être (Deloria 1992).

Ce n'est que sous l'influence du christianisme et, après coup, dans le contexte du développement du mouvement pan-indien, que les diverses cultures amérindiennes auraient commencé à désigner l'Être suprême au moyen de noms plus "personnalisés " ou "familiers " tels que Grand Esprit, Gitchi-Manitou, Createur, Pere, Grand-Pere (Powers 1975; Brown 1990). Mais cette évolution ne semble pas avoir affecté fondamentalement leur vision et leur expérience de l'Être suprême. Le nom primitif, Wakan Tanka, demeure donc un point de repère très valable pour évaluer la conception amérindienne de l'Être suprême, même chez les autochtones qui ont adopté le christianisme. L'Être suprême est perçu comme comme présent dans tous les éléments de l'univers, tout en étant une réalité 
distincte. Selon les Lakota, il posséde la capacité d'être en même temps "un" et "tout" ou d'être "plusieurs-en-un-seul". Certains le perçoivent donc comme une "personne" distincte qui est le "Grand-Père" de tous les êtres. D'autres l'expérimentent davantage comme une "présence» ou comme une "puissance» (Stolzman 1986: 181). William Powers, un des grands experts de la religion Oglala, affirme, de son côté, que le terme Wakan Tanka est singulier dans sa forme mais collectif dans son sens. Wakan Tanka, comme tel, n'est pas personnifié. Seulement certains aspects de Wakan Tanka, le sont, comme, par exemple, le soleil, la lune, le ciel, les vents, les éclairs, le tonnerre, où d'autres phénomènes que les Lakota associent spontanément avec l'Être suprême (Powers 1975: 45-46).

Cette dernière intuition nous offre aussi un point de repère valable pour comprendre comment les Amérindien(ne)s conçoivent le rapport entre l'Être suprême et les entités qu'ils/elles désignent comme "esprits". Les traditions autochtones, orales ou écrites, offrent peu d'informations précise sur cette question qui est souvent perçue comme une question typiquement "occidentale" à cause de la conception juive et chrétienne de Dieu comme un Être suprême "personnalisé". Si, pour les Amérindien(ne)s, Dieu est en même temps «un» et «tout », les «esprits » font partie de la réalité intime de l'Être suprême tout en étant distincts de lui. Les "esprits" sont perçus comme des puisances, des énergies, des agents par qui l'Être suprême agit dans le monde. Ils sont des manifestations concrètes du Grand Mystère. Ils appartiennent au « royaume » intime de l'Être suprême, où se trouvent aussi l'âme ou l'esprit des personnes qui sont décédées, mais ont aussi la capacité d'agir dans l'univers des humains.

Nous constatons immédiatement que dans notre approche de cette dimension mystérieuse de la réalité amérindienne nous sommes victimes du concept de "personne" qui se trouve au centre de notre approche occidentale et chrétienne de Dieu. Nous devons reconnaître que le concept de "personne", sur lequel est fondée la vision monothéiste de Dieu, fonctionne difficilement dans les religions dites "cosmiques", "naturelles" ou "primaires", telles que les religions amérindiennes. Dans l'étude remarquable qu'il consacre à l'identité religieuse des Oglala Lakota, Paul Steinmetz affirme que le "monothéisme explicite" relève d'un type de connaissance conceptuelle qui est le produit d'un processus philosophique que nous ne retrouvons pas dans les cultures où domine la pensée symbolique. Dans ces cultures, il n'existe qu'un «monothéisme implicite» (Steinmetz 1990: 39-40). Il se produit une certaine tension entre la "tradition supérieure" (représentée par les chefs spirituels, les sages) et la «tradition inférieure» (le peuple) au sein de ces cultures religieuses quand elles entrent en contact avec le christianisme. Cette tension concerne 
la compréhension même de l'Être suprême. Afin d'éviter que les religions amérindiennes ne soient perçues comme des religions «animistes" (ce qu'elles ne sont pas!), la tradition supérieure tend à accentuer le caractère absolument mystérieux et "surnaturel" de l'Être suprême, alors que la tradition inférieure, surtout au niveau des pratiques et des rituels religieux, ne craint pas d'affirmer l'existence des puissances spirituelles qui sont les manifestations concrètes du Grand Mystère (Schwarz 1981: 51-58). Nos rencontres personnelles avec un certain nombre de représentants de la «tradition supérieure " chez les Cris et les Anishnabe, nous ont aidé à mieux comprendre la fragilité du concept analogique de personne, une fois qu'il est appliqué à l'Être suprême. Les conceptions spécifiquement amérindiennes de Dieu, tout comme la mystique de l'advaita (voir Dupuis 1989: 311), représentent une approche unique de la transcendance et de l'immanence de l'Être surprême dont le christianisme pourrait profiter énormément pour dépasser sa conception dualiste de Dieu ET le monde.

\section{L'actualité des religions amérindiennes}

\subsection{L'accès au monde des esprits}

Les réflexions précédentes ont montré que dans l'univers contemporain des Amérindien(ne)s les esprits sont toujours omniprésents. Nous devons cependant constater que la littérature "scientifique" sur cette dimension importante de la réalité amérindienne demeure remarquablement pauvre. Lors du colloque sur «La symbolique de l'esprit et du pouvoir chez les peuples autochtones de l'Amérique du Nord" qui s'est tenu à l'Université d'Ottawa en octobre 1993, nous avons pu constater les limites des recherches anthropologiques dans ce domaine. Il est intéressant de noter qu'un certain nombre d'anthropologues sont venus à la conclusion que ce fait n'est pas dû simplement à l'incompétence linguistique des chercheurs (connaissance des langues autochtones), mais à leur incapacité d'entrer de façon personnelle et expérientielle dans l'univers spirituel des Amérindien(ne)s. L'inculturation de la grande majorité des anthropoloques, des théologien(ne)s et des experts en sciences religieuses est fondamentalement différente des celle des Amérindien(ne)s. Généralement, ils ne partagent pas les mêmes modes d'apprentissage (par exemple, les rêves et visions) et les mêmes types de connaissances (par exemple, les connaissances expérientielles de nature mystique). De là leur difficulté d'entrer dans le processus cognitif qui forme le substratum de l'expérience amérindienne et de saisir l'unicité de cette expérience. Dans le domaine des religions ou des spiritualités d'orientation mystique, comme les spiritualités amérindiennes, rien ne remplace 
l'expérience personnelle (Starkloff 1985). Il ne suffit pas ici de se contenter d'un simple regard objectif ou d'une approche théorique. La "voie initiatique" est ici inévitable. C'est cette voie que nous avons essayé de pratiquer entre 1983 et 1987 lors d'une série de rencontres avec des Indiens cris de l'Alberta (Peelman 1991). Quiconque s'engage aujourd'hui dans une telle démarche se heurte au scepticisme et au dédain. Tout comme il fut naguère impossible pour un Rudolf Bultmann "d'utiliser l'éclairage électrique et la radio, d'employer en cas de maladie les moyens médicaux et cliniques, et de croire en même temps au monde d'esprits et de miracles dont parle le Nouveau Testament" (Bultmann 1955: 143), il est difficile d'apprécier ce que les «esprits » viennent faire dans la vie d'un autochtone dont la maison est équipée d'une antenne parabolique qui peut capter 150 postes de télévision! Quiconque entre aujourd'hui dans l'univers amérindien doit être capable d'accueillir des "expériences extraordinaires» qui bouleversent ses attitudes et connaissances acquises. 11/elle doit accepter de "se laisser transformer" par ces expériences afin de comprendre que notre monde (la «réalité») est plus complexe que sa perception actuelle selon les canons de la culture sécularisée et technologique de l'Occident (Young et Goulet 1994).

\subsection{L'inconscient collectif de l'humanité}

Les anthropologues et les théologien(ne)s doivent cependant faire face à la tâche difficile de traduire dans un langage académique les résultats des "expériences extraordinaires" qu'ils/elles ont vécues dans l'univers spirituel des Amérindien(ne)s. Il n'y a pas de consensus à propos de cette démarche. En ce qui concerne les entités que les autochtones désignent comme "esprits", il nous semble qu'on peut se fier aux rercherches de Carl Jung à propos de l'inconscient collectif de l'humanité. En étudiant ce phénomène, Jung a établi des parallèles avec les croyances primitives dans les âmes et les esprits. Les âmes appartiennent aux complexes autonomes de l'inconscient individuel, tandis que les esprits appartiennent aux complexes autonomes de l'inconscient collectif. Tenant compte du rôle extraordinaire que la croyance dans les esprits a joué au cours de l'histoire, Jung refuse de considérer les esprits comme des «êtres bizarres" que les sciences modernes devraient mettre de côté. Les esprits peuvent être des fantaisies pathologiques qui sont expérimentées (dangereusement) par des personnes déconnectées de la réalité, mais ils peuvent aussi représenter des idées nouvelles dont on ne connaît pas encore la portée (Jung 1972: 301-318). C'est dans ce dernier sens que nous sommes tentés d'interpréter les esprits amérindiens en tant qu'ils représentent une dimension oubliée ou inconnue (par nous) de la réalité. Au 
cours des siècles, les traditions spirituelles des Amérindien(ne)s ont réussi à maintenir et à développer une approche unique du monde et de la réalité : un «mysticisme naturel » de haute qualité. Alors que le christianisme est marqué par une conscience aiguë de l'histoire et les grandes religions de l'Asie par celle de la transcendance divine, les religions amérindiennes se distinguent par une spiritualité holistique qui affirme le caractère sacré de l'univers et de tous les êtres qui y habitent. Cette spiritualité affirme un lien indissoluble entre le cosmique, l'humain et le divin. Serait-il possible de considérer les "esprits» comme des manifestations concrètes de ce réseau inclusif de relations? Thomas Berry du Riverdale Center for Religious Research parle d'un "numinous presence" et d'un "numinous mode of consciousness" dont la signification concerne l'avenir de toute l'humanité (Berry 1976:136). Les religions amérindiennes représentent des expériences primordiales que nous pouvons considérer comme des révélations divines et comme une dimension constitutive et irremplaçable de l'être humain. Elles constituent le fondement même des cultures amérindiennes et de la structure psychique de ses membres. En même temps, elles ont une signification universelle qui mériterait plus d'attention au niveau des recherches interculturelles. Joseph Epes Brown, réfléchissant sur les trois dimensions (purification, expansion, union) du processus spirituel, confirme ce point de vue quand il affirme:

Il nous faut cesser d'être une partie, un fragment imparfait, et comprendre ce que nous sommes vraiment afin d'élargir notre conscience jusqu'à inclure en nous l'univers. C'est seulement alors, lorsque ces deux conditions de purification et d'expansion seront réalisées, que nous pourrons atteindre l'étape ultime de l'union. Toutes les religions majeures attestent que la plus grave erreur commise par l'homme (sic) est de considérer le vrai soi comme n'étant que le corps ou l'esprit. Or c'est seulement en passant par des disciplines traditionnelles comme celles des Indiens des Plaines que l'homme (sic) pourra dissiper cette grande illusion (Brown 1990: 72).

\subsection{La valeur thérapeutique des rituels amérindiens}

Pour conclure cette excursion dans l'univers spirituel des Amérindien(ne)s il convient d'insister brièvement sur la réévaluation de leur médecine traditionnelle. On reconnaît désormais que les traumatismes dont les Amérindien(ne)s souffrent et meurent aujourd'hui ne sont pas d'abord physiques ou psychologiques, mais d'ordre culturel. Ils sont liés à la déculturation graduelle de beaucoup de communautés autochtones. Face à cette situation dramatique, l'attention se porte de plus en plus vers la médecine traditionnelle, les attitudes ancestrales vis-à-vis la 
santé et la maladie, et certains rituels de guérison qui possèdent peut-être de plus grandes vertus curatives que les thérapies psychologiques modernes. Les ancien(ne)s affirment que la santé est le résultat de l'équilibre entre le physique, le mental et le spirituel, tandis que la maladie est souvent le résultat d'un manque d'équilibre entre ces trois éléments ou de la rupture des relations vitales, même si certaines maladies sont causées par des facteurs externes à la personne.

Le travail du médecin et psychiatre Wolfgang Jilek et de son épouse Louise Jilek-Aall (psychiatre et anthropologue) auprès de certains groupes autochtones de la Colombie-Britannique mérite d'être mentionné ici. Leur recherche sur le terrain en collaboration étroite avec des guérisseurs traditionnels et des chamanes confirme la crédibilité et la valeur thérapeutique de certains rituels ancestraux tels que la Danse de l'Esprit (Jilek 1982). Un peu partout au Canada, on reconnaît graduellement le besoin de thérapies interculturelles qui impliquent des médecins autochtones et des chamans, et qui tiennent compte de l'univers spirituel des Amérindien(ne)s. Entre 1984 et 1989, une équipe de chercheur(e)s de l'Université de l'Alberta s'est engagée dans un programme de traitement de la psoriasis avec Russell Willier, un médecin traditionnel de Sucker Creek en Alberta. Le traitement, dont les résultats positifs sont scientifiquement établis, impliquait la suerie et des références explicites à l'univers spirituel des Amérindien(ne)s (Young 1989). Depuis quelques années, on voit émerger des centres, tel que le Nechi Institute d'Edmonton (Hodgson 1989), qui favorisent un processus de guérison holistique, maintenant un lien solide entre les patients et leurs communautés culturelles. En 1985, le Service correctionnel du Canada approuvait une politique sur la spiritualité amérindienne permettant aux prisonniers autochtones d'avoir accès à leurs chefs spirituels, ancien(ne)s, guérisseurs et chamanes. Ceux-ci peuvent désormais faire partie des services d'aumôniers au même titre que les ministres des diverses Églises chrétiennes et des religions reconnues (Peelman 1986). Une analyse récente de l'application de cette politique a conclu que la spiritualité amérindienne peut être considérée comme un exemple de "guérison symbolique" pour qui le concept de "sacré" (sainteté/santé) est fondamental pour la compréhension du processus de guérison (Waldram 1993).

Pratiquement toutes les thérapies amérindiennes s'inspirent de l'enseignement traditionnel de la "roue médicinale" (Medicine Wheel). Cet enseignement varie d'un groupe autochtone à l'autre, mais son symbolisme est devenu pan-indien. La roue ou le cercle symbolise l'ensemble de l'univers et toutes les puissances spirituelles ou "relations" qui s'y trouvent. Ces puissances constituent le réseau de relations dont chaque être 
vivant a besoin pour son développement harmonieux et pour entrer ou demeurer dans le «cercle sacré de la vie». Certains groupes autochtones continuent à "personnifier» ces forces spirituelles en les associant avec des esprits-animaux. Par exemple, la cosmologie sacrée de Russell Willier comprend l'esprit du buffalo (Nord), l'esprit de l'aigle (Est), l'esprit de la souris (Sud) et l'esprit de l'ours (Ouest) (Young 1992: 24). D'autres groupes autochtones y voient plutôt un ensemble de valeurs, d'attitudes, d'aptitudes et de comportements dont l'être humain a besoin pour son développement intégral selon les divers âges de la vie. Le symbole de la "roue médicinale" sert de miroir pour la personne humaine qui se met en route afin d'accomplir son existence de façon harmonieuse en tenant compte de toutes les "relations" qui lui viennent en aide. Il importe peu si les divers dons qui se trouvent dans les quatre points cardinaux de l'univers, dans la terre-mère et au ciel soient perçus comme des "entités spirituelles" (esprits) ou comme des "valeurs". La thérapie de la "roue médicinale" est disponible aussi bien pour les Amérindien(ne)s qui maintiennent une conception religieuse de l'univers que pour ceux et celles qui ont adopté une vision sécularisée du monde. La "roue médicinale» accentue l'unicité de chaque personne et l'universalité des dons qui sont partagés par l'ensemble de l'humanité (Four Worlds 1984).

\section{Conclusion}

La vision du monde que nous retrouvons aujourd'hui chez beaucoup de groupes amérindiens a subi l'influence du christianisme et l'impact de la civilisation occidentale, mais elle n'a pas été fondamentalement ébranlée par ce processus d'acculturation. Cette cosmologie sacrée continue à échapper à notre contrôle scientifique et même à notre compréhension émotionnelle. De fait, nous sommes confrontés ici avec des «expériences extraordinaires" où les frontières entre ce que nous appelons "nature " et "surnaturel" sont pratiquement inexistantes. Que penser, par exemple, de la prière que les Indiens koyukon de l'Alaska adressent à l'esprit du corbeau (Nelson 1983)? Que penser du lien mystique que le chasseur innu du Québec et du Labrador entretient avec l'esprit du caribou qu'il veut capturer au moyen de son chant et du battement de son tambour? Que penser de l'attitude religieuse des Cri et des Anishnabe devant les os des animaux qu'ils ont tués, afin d'apaiser leur esprit et de maintenir de bonnes relations avec eux (Tanner 1979)? Que penser de tous ces Amérindien(ne)s, parfois très acculturé(e)s, qui pratiquent le Vision quest, afin de rencontrer leur "esprit gardien» qui se manifeste souvent sous la forme d'un esprit animal (Amiotte 1976)? Nous pouvons multiplier ici les exemples qui illustrent que dans l'univers contemporain des 
Amérindien(ne)s les esprits ont toujours leur place. À moins de se contenter de "tourisme ésotérique", les non-autochtones qui s'aventurent dans cet univers, à la fois si proche et si loin de nous, doivent apprendre à "danser avec les esprits" (Ross 1992) et à voir avec "un œil amérindien" (Capps 1976), s'ils/elles veulent communier en profondeur à la "conscience» ou à la "puissance spirituelle" dont l'environnement amérindien demeure investi. 


\section{Bibliographie}

AMIOTTE, A. 1976. «Eagles Fly Over», Parabola. Myth and Quest for Meaning 1/3: 28-41. Également dans Native American Traditions. Sources and Interpretations. (GILL, S.). Belmont, Wadsworth Publishing Company, p. 90-104.

BERRY, T. 1976. "The Indian Future", Cross Currents $26: 133-142$.

BROWN, J.E. 1971. The Sacred Pipe. Black Elk's Account of the Seven Rites of the Oglala Sioux. Baltimore, Penguin Books.

BROWN, J.E. 1990. L'héritage spirituel des Indiens d'Amérique. Éd. Le Mail. Traduction de: The Spiritual Legacy of the American Indian. New York, Crossroad.

BULTMANN, R. 1955. L'interpretation du Nouveau Testament. Paris, Aubier.

CAPPS, W. (éd.). 1976. Seeing with a Native Eye. San Francisco, Harper \& Row.

DELORIA, V. 1992. God is Red. A Native View of Religion. Second Edition. G, Colorado, North American Press.

DUPUIS, J. 1989. Jésus-Christ à la rencontre des religions. Paris, Desclée. EVANS-PRITCHARD, E. 1956. Theories of Primitive Religion. London, Oxford University Press.

FOUR WORLDS. 1984. The Sacred Tree. Lethbridge, Four Worlds Development Press, The University of Lethbridge.

GILL, S. 1987. Native American Religious Action. A Performative Approach to Religion. Columbia, University of South Dakota Press.

GOULET, J.-G., 1992. "Visions et conversions chez les Déné Tha. Expériences religieuses chez un peuple autochtone converti", Religiologiques 6: 147-182.

HODGSON, M. 1989. "The Eagle Has Landed », Kerygma $23: 195-203$.

JILEK, W. 1982. Indian Healing. Shamanic Ceremonialism in the Pacific Northwest Today. Surrey-Washington, Hancock House.

JUNG, C. 1972. The Structure and Dynamics of the Psyche. (Collected Works, 8), London, Routledge and Kegan Paul. 
LAROQUE, E. 1975. Defeathering the Indian. Agincourt, The Book Society of Canada.

MARTY, M.E. 1990. "Foreword", dans Nature Religion in America. From the Algonkian Indians to the New Age. (ALBANESE, C.L. ed.). Chicago, The Uiversity of Chicago Press.

NELSON, R. 1983. Make Prayers to the Raven. A Koyukon View of the Northern Forest. Chicago, The University of Chicago Press.

PEELMAN, A. 1986. «Traditional Native Spiritual Practice in Canada's Federal Prisons ", Kerygma 20: 101-109.

PEELMAN, A. 1991. "Christianisme et cultures amérindiennes. Présentation et analyse d'une démarche théologique interculturelle», ÉglThéol 22: 131-156.

PEELMAN, A. 1992. Le Christ est amérindien. Une réflexion théologique sur l'inculturation $d u$ Christ parmi les Amerindiens $d u$ Canada. Ottawa, Novalis / Université Saint-Paul.

POWERS, W. 1975. Oglala Religion. Lincoln, University of Nebraska Press.

ROSS, R. 1992. Dancing with a Ghost. Exploring Indian Reality. Markham, Octopus Publishing Group.

SCHLIER, H. 1958. Mächte und Gewalten im Neuen Testament. Freiburg, Herder.

SCHLIER, H. 1968. Essais sur le Nouveau Testament. Paris, Cerf.

SCHWARZ, D. 1981. Plains Indian Theology: As Expressed in Myth and Ritual, and in the Ethics of the Culture. Ann Arbor, University Microfilms International.

STARKLOFF, C. 1985. «Religious Renewal in Native North America: A Contemporary Call to Mission", Missiology. An International Review $13: 81-101$.

STEINMETZ, P. 1990. Pipe, Bible and Peyote Among the Oglala Lakota. A Study in Religious Identity. Knoxville, The University of Tennessee Press.

STOLZMAN, W. 1986. The Pipe and Christ. A Christian-Sioux Dialogue. Camberlain, South Dakota, Tipi Press. 
TANNER, A. 1979. Bringing Home Animals. Religious Ideology and Mode of Production of the Mistassini Cree Hunters. New York, St. Martin's Press; London, C. Hurst.

WALDRAM, J. 1993. «Aboriginal Spirituality: Symbolic Healing in Canadian Prisons", Culture, Medicine and Psychiatry 17: 345-362.

WINK, W. 1984. Naming the Powers. The Language of Power in the New Testament. Philadelphia, Fortress Press.

WINK, W. 1986. Unmasking the Powers. The Invisible Forces That Determine Human Existence. Philadelphia, Fortress Press.

YOUNG, D., INGRAM, G., SWARTZ, L. 1989. Cry of the Eagle. Encounters with a Cree Healer. Toronto, University of Toronto Press.

YOUNG, D. et J.-G. GOULET (éd.). 1994. Being Changed by CrossCultural Encounters. The Anthropology of Extraordinary Experience. Peterborough, Broadview Press. 